\title{
Research Article \\ Complete Convergence for Maximal Sums of Negatively Associated Random Variables
}

\author{
Victor M. Kruglov \\ Department of Statistics, Faculty of Computational Mathematics and Cybernetics, \\ Moscow State University, Vorobyovy Gory, GSP-1, 119992, Moscow, Russia \\ Correspondence should be addressed to Victor M. Kruglov, krugvictor@gmail.com
}

Received 24 December 2009; Accepted 1 April 2010

Academic Editor: Mohammad Fraiwan Al-Saleh

Copyright (C) 2010 Victor M. Kruglov. This is an open access article distributed under the Creative Commons Attribution License, which permits unrestricted use, distribution, and reproduction in any medium, provided the original work is properly cited.

Necessary and sufficient conditions are given for the complete convergence of maximal sums of identically distributed negatively associated random variables. The conditions are expressed in terms of integrability of random variables. Proofs are based on new maximal inequalities for sums of bounded negatively associated random variables.

\section{Introduction}

The paper by Hsu and Robbins [1] initiated a great interest to the complete convergence of sums of independent random variables. Their research was continued by Erdös [2, 3], Spitzer [4], and Baum and Katz [5]. Kruglov et al. [6] proved two general theorems that provide sufficient conditions for the complete convergence for sums of arrays of row-wise independent random variables. In the paper of Kruglov and Volodin [7], a criterion was proved for the complete convergence of sums of independent identically distributed random variable in a rather general setting. Taylor et al. [8] and Chen et al. $[9,10]$ demonstrated that many known sufficient conditions for complete convergence of sums of independent random variables can be transformed to sufficient conditions for the complete convergence of sums of negatively associated random variables. Here we give necessary and sufficient conditions for the complete convergence of maximal sums of negatively associated identically distributed random variables. They resemble the criterions presented by Baum and Katz [5] and by Kruglov and Volodin [7] for the complete convergence of sums of independent identically distributed random variables. Theorems 2.3 and 2.5 are our main results. Theorem 2.3 is new even for independent random variables. 
In what follows we assume that all random variables under consideration are defined on a probability space $(\Omega, \mathcal{F}, P)$. We use standard notations, in particular, $I_{A}$ denotes the indicator function of a set $A \subseteq \Omega$. Recall the notion of negatively associated random variables and some properties of such random variables.

Definition 1.1. Random variables $X_{1}, \ldots, X_{n}$ are called negatively associated if

$$
\operatorname{Cov}\left(f\left(X_{i_{1}}, \ldots, X_{i_{k}}\right), g\left(X_{j_{1}}, \ldots, X_{j_{m}}\right)\right) \leq 0
$$

for any pair of nonempty disjoint subsets $A=\left\{i_{1}, \ldots, i_{k}\right\}$ and $B=\left\{j_{1}, \ldots, j_{m}\right\}, k+m \leq n$, of the set $\{1, \ldots, n\}$ and for any bounded coordinate-wise increasing real functions $f\left(x_{i_{1}}, \ldots, x_{i_{k}}\right)$ and $g\left(x_{j_{1}}, \ldots, x_{j_{m}}\right), x_{1}, \ldots, x_{n} \in \mathbb{R}=(-\infty, \infty)$. Random variables $X_{n}, n \in \mathbb{N}=\{1,2, \ldots$,$\} are$ negatively associated if for any $n \in \mathbb{N}$ random variables $X_{1}, \ldots, X_{n}$ are negatively associated.

In this definition the coordinate-wise increasing functions $f$ and $g$ may be replaced by coordinate-wise decreasing functions. Indeed, if $f$ and $g$ are coordinate-wise decreasing functions, then $-f$ and $-g$ are coordinate-wise increasing functions and the covariance (1.1) coincides with the covariance for $-f$ and $-g$.

Theorem A. Let $X_{n}, n \in \mathbb{N}$, be negatively associated random variables. Then for every $a_{n}, b_{n} \in$ $\mathbb{R}, a_{n} \leq b_{n}$, the random variables $Y_{n}=a_{n} I_{\left(-\infty, a_{n}\right)}\left(X_{n}\right)+X_{n} I_{\left[a_{n}, b_{n}\right]}\left(X_{n}\right)+b_{n} I_{\left(b_{n}, \infty\right)}\left(X_{n}\right), n \in \mathbb{N}$, are negatively associated. For every $n \in \mathbb{N}$ and $x_{1}, \ldots, x_{n} \in \mathbb{R}$, the inequalities

$$
\begin{aligned}
& P\left\{X_{1} \leq x_{1}, \ldots, X_{n} \leq x_{n}\right\} \leq \prod_{k=1}^{n} P\left\{X_{k} \leq x_{k}\right\}, \\
& P\left\{X_{1} \geq x_{1}, \ldots, X_{n} \geq x_{n}\right\} \leq \prod_{k=1}^{n} P\left\{X_{k} \geq x_{k}\right\}
\end{aligned}
$$

hold.

Proof. It can be found in Taylor et al. [8].

Theorem B. Let $X_{n}$ be negatively associated random variables. Let $X_{n}^{*}, n \in \mathbb{N}$, be independent random variables such that $X_{k}$ and $X_{k}^{*}$ are identically distributed for every $k=1, \ldots, n$. Then

$$
E \exp \left\{X_{1}+\cdots+X_{n}\right\} \leq E \exp \left\{X_{1}^{*}+\cdots+X_{n}^{*}\right\} .
$$

If $E\left|X_{k}\right|^{p}<\infty$ and $E X_{k}=0$ for all $k=1, \ldots, n$ and for some $p \geq 1$, then

$$
E\left|\max _{1 \leq r \leq n} \sum_{k=1}^{r} X_{k}\right|^{p} \leq 2 E\left|\sum_{k=1}^{n} X_{k}^{*}\right|^{p}, \quad n \in \mathbb{N} .
$$

Proof. It can be found in Qi-Man Shao [11]. 


\section{Main Results}

Our basic theorems will be stated in terms of special functions. They were introduced in Kruglov and Volodin [7].

Definition 2.1. A nonnegative function $h(x), x \in[0, \infty)$, belongs to the class $\mathscr{d}_{q}$ for some $q \in(0,2)$ if it is nondecreasing, is not equal to zero identically, and

$$
\begin{gathered}
\limsup _{x \rightarrow \infty} \frac{h(2 x)}{h(x)}<\infty, \\
\limsup _{y \rightarrow \infty} \frac{y^{2 / q-1}}{h(y)} \int_{y}^{\infty} h(x) x^{-2 / q} d x<\infty .
\end{gathered}
$$

The class $\mathscr{\ell}_{q}$ contains all nondecreasing nonnegative functions slowly varying at infinity which are not equal to zero identically, and in particular, $\ln ^{\beta}(1+x)$ with $\beta>0$. The functions $x^{\alpha}$ and $x^{\alpha} \ln ^{\beta}(1+x)$ with $\alpha \in[0,2 / q-1)$ and $\beta>0$ are also in $\mathscr{L}_{q}$.

Remark 2.2. If a nonnegative function $h(x), x \in[0, \infty)$, is nondecreasing and satisfies condition (2.1), then

$$
h(x) \leq \Delta x^{l}
$$

for all $x$ greater than some $x_{0} \geq 1$ and for some $\Delta>0$ and $l>0$.

Proof. We may assume that $h(x)>0$ for all $x$ greater than some $x_{0} \geq 1$. From condition (2.1), it follows that $\sup _{x \geq x_{0}} h(2 x) / h(x)=d<\infty$. Choose a number $l>0$ such that $d \leq 2^{l}$. If $x \geq 2 x_{0}$, then $2^{n} x_{0} \leq x<2^{n+1} x_{0}$ for some $n \in \mathbb{N}$ and $h(x) \leq h\left(2^{n+1} x_{0}\right) \leq d^{n+1} h\left(x_{0}\right) \leq 2^{l(n+1)} h\left(x_{0}\right) \leq$ $2^{l} x^{l} h\left(x_{0}\right)$. Inequality (2.3) holds for all $x \geq x_{0}$ with $\Delta=2^{l} h\left(x_{0}\right)$.

Theorem 2.3. Let $X_{n}, n \in \mathbb{N}$, be negatively associated identically distributed random variables, $S_{n}=$ $X_{1}+\cdots+X_{n}, 0<q<2, r>1$. Let $h(x), x \in[0, \infty)$, be a function which is nondecreasing, is not equal to zero identically, and satisfies condition (2.1). Then the following conditions are equivalent:

$$
\begin{aligned}
& E\left(\left|X_{1}\right|^{r q} h\left(\left|X_{1}\right|^{q}\right)\right)<\infty, \quad E X_{1}=0, \quad \text { for } q \geq 1, \\
& \sum_{n=1}^{\infty} n^{r-2} h(n) P\left\{\max _{1 \leq k \leq n}\left|S_{k}\right|>\varepsilon n^{1 / q}\right\}<\infty, \quad \forall \varepsilon>0, \\
& \sum_{n=1}^{\infty} n^{r-2} h(n) P\left\{\sup _{k \geq n}\left|k^{-1 / q} S_{k}\right|>\varepsilon\right\}<\infty, \quad \forall \varepsilon>0 .
\end{aligned}
$$


Corollary 2.4. Let $X_{n}, n \in \mathbb{N}$, be negatively associated identically distributed random variables, $S_{n}=$ $X_{1}+\cdots+X_{n}, 0<q<2, r>1, \beta \geq 0$. The following conditions are equivalent:

$$
\begin{gathered}
E\left(\left|X_{1}\right|^{r q}\left(\ln \left(1+\left|X_{1}\right|\right)\right)^{\beta q}\right)<\infty, \quad E X_{1}=0, \quad \text { for } q \geq 1, \\
\sum_{n=1}^{\infty} n^{r-2}(\ln n)^{\beta} P\left\{\max _{1 \leq k \leq n}\left|S_{k}\right|>\varepsilon n^{1 / q}\right\}<\infty, \quad \forall \varepsilon>0, \\
\sum_{n=1}^{\infty} n^{r-2}(\ln n)^{\beta} P\left\{\sup _{k \geq n}\left|k^{-1 / q} S_{k}\right|>\varepsilon\right\}<\infty, \quad \forall \varepsilon>0 .
\end{gathered}
$$

A part of Theorem 2.3 can be generalized to a larger range of $r, r \geq 1$, under additional restrictions on functions $h(x), x \in[0, \infty)$.

Theorem 2.5. Let $X_{n}, n \in \mathbb{N}$, be negatively associated identically distributed random variables, $S_{n}=$ $X_{1}+\cdots+X_{n}, h \in \mathfrak{l}_{q}, 0<q<2, r \geq 1$. Then the following conditions are equivalent:

$$
\begin{aligned}
& E\left(\left|X_{1}\right|^{r q} h\left(\left|X_{1}\right|^{q}\right)\right)<\infty, \quad E X_{1}=0, \quad \text { for } q \geq 1, \\
& \sum_{n=1}^{\infty} n^{r-2} h(n) P\left\{\max _{1 \leq k \leq n}\left|S_{k}\right|>\varepsilon n^{1 / q}\right\}<\infty, \quad \forall \varepsilon>0 .
\end{aligned}
$$

Corollary 2.6. Let $X_{n}, n \in \mathbb{N}$, be negatively associated identically distributed random variables, $S_{n}=$ $X_{1}+\cdots+X_{n}, 0<q<2, \beta \geq 0$. The following conditions are equivalent:

$$
\begin{gathered}
E\left(\left|X_{1}\right|^{q}\left(\ln \left(1+\left|X_{1}\right|\right)\right)^{\beta q}\right)<\infty, \quad E X_{1}=0, \quad \text { for } q \geq 1, \\
\sum_{n=1}^{\infty} \frac{(\ln n)^{\beta}}{n} P\left\{\max _{1 \leq k \leq n}\left|S_{k}\right|>\varepsilon n^{1 / q}\right\}<\infty, \quad \forall \varepsilon>0 .
\end{gathered}
$$

Proof of Theorem 2.3. The theorem is obvious if the random variable $X_{1}$ is degenerate, that is, $P\left\{X_{1}=\right.$ const. $\}=1$. From now on we suppose that the random variable $X_{1}$ is not degenerate. Denote

$$
\delta= \begin{cases}\frac{r-1}{r+l} & \text { if } 0<r q<1, \\ \frac{r-1}{4(r+l)} & \text { if } 1 \leq r q \leq 2, \\ \frac{2-q}{4 q(r+l)} & \text { if } r q>2\end{cases}
$$


where $l$ is the same as in (2.3). Define the function $f(x), x \in \mathbb{R}$,

$$
f(x)= \begin{cases}\varepsilon n^{1 / q} \delta & \text { if } x>\varepsilon n^{1 / q} \delta \\ x & \text { if }|x| \leq \varepsilon n^{1 / q} \delta \\ -\varepsilon n^{1 / q} \delta & \text { if } x<-\varepsilon n^{1 / q} \delta\end{cases}
$$

where $\varepsilon>0$ is a fixed number. By Theorem A the random variables $Y_{k}=f\left(X_{k}\right), k=1, \ldots, n$, are negatively associated. Put $S_{k}^{\prime}=Y_{1}+\cdots+Y_{k}, k=1, \ldots, n$. Note that $\left|Y_{1}\right| \leq\left|X_{1}\right|$ and $Y_{1} \rightarrow X_{1}$ as $n \rightarrow \infty$. If $E\left|X_{1}\right|^{r q}<\infty$, then by the dominated convergence theorem we have

$$
\begin{gathered}
\lim _{n \rightarrow \infty} E\left|Y_{1}\right|^{r q}=E\left|X_{1}\right|^{r q}, \\
\lim _{n \rightarrow \infty} E\left|Y_{1}-E Y_{1}\right|^{r q}=E\left|X_{1}-E X_{1}\right|^{r q} \quad \text { if } r q \geq 1 .
\end{gathered}
$$

Prove that $(2.4) \Rightarrow(2.5)$. Assume that $0<r q<1$. The probability $P\left\{\max _{1 \leq k \leq n}\left|S_{k}\right|>\varepsilon n^{1 / q}\right\}$ can be estimated as follows:

$$
\begin{aligned}
P\left\{\max _{1 \leq k \leq n}\left|S_{k}\right|>\varepsilon n^{1 / q}\right\}= & P\left\{\left\{\max _{1 \leq k \leq n}\left|S_{k}^{\prime}\right|>\varepsilon n^{1 / q}\right\} \cap \bigcap_{k=1}^{n}\left\{X_{k}=Y_{k}\right\}\right\} \\
& +P\left\{\left\{\max _{1 \leq k \leq n}\left|S_{k}\right|>\varepsilon n^{1 / q}\right\} \cap\left(\bigcup_{k=1}^{n}\left\{X_{k} \neq Y_{k}\right\}\right)\right\} \\
& \leq P\left\{\max _{1 \leq k \leq n}\left|S_{k}^{\prime}\right|>\varepsilon n^{1 / q}\right\}+P\left\{\max _{1 \leq k \leq n}\left|X_{k}\right|>\varepsilon n^{1 / q} \delta\right\} .
\end{aligned}
$$

We intend to use Lemma 3.1 from the third part of the paper. Put $\gamma=r q, x=\varepsilon n^{1 / q}, c=$ $\varepsilon n^{1 / q}(r-1) /(r+l)$. From (2.13) it follows that the inequality $E\left|Y_{1}\right|^{\gamma}<2 E\left|X_{1}\right|^{\gamma}$ holds for all $n \in \mathbb{N}$ greater than some $n_{0}$. By inequality (3.1), we get, for all $n>n_{0}$,

$$
\begin{aligned}
P\left\{\max _{1 \leq k \leq n}\left|S_{k}^{\prime}\right|>\varepsilon n^{1 / q}\right\} & \leq 2 \exp \left\{\frac{x}{c}-\frac{x}{c} \ln \left(\frac{x c^{\gamma-1}}{n E\left|Y_{1}\right|^{r}}+1\right)\right\} \\
& \leq 2 \exp \left\{\frac{r+l}{r-1}-\frac{r+l}{r-1} \ln \left(\frac{\varepsilon^{r q}(r-1)^{r q-1}}{2 E\left|X_{1}\right|^{r q}(r+l)^{r q-1}} n^{r-1}+1\right)\right\} \\
& \leq 2 \exp \left\{\frac{r+l}{r-1}\right\}\left(\frac{\varepsilon^{r q}(r-1)^{r q-1}}{2 E\left|X_{1}\right|^{r q}(r+l)^{r q-1}}\right)^{-(r+l) /(r-1)} n^{-(r+l)}=C n^{-(r+l)} .
\end{aligned}
$$

These inequalities and (2.3) imply that

$$
\sum_{n=n_{0}+1}^{\infty} n^{r-2} h(n) P\left\{\max _{1 \leq k \leq n}\left|S_{k}^{\prime}\right|>\varepsilon n^{1 / q}\right\} \leq \Delta C \sum_{n=n_{0}+1}^{\infty} n^{r+l-2} n^{-(r+l)}<\infty
$$


Since $P\left\{\max _{1 \leq k \leq n}\left|X_{k}\right|>\varepsilon n^{1 / q} \delta\right\} \leq n P\left\{\left|X_{1}\right|>\varepsilon n^{1 / q} \delta\right\}$, we obtain

$$
\begin{aligned}
\sum_{n=n_{0}+1}^{\infty} n^{r-2} h(n) P\left\{\max _{1 \leq k \leq n}\left|X_{k}\right|>\varepsilon n^{1 / q} \delta\right\} & \leq \sum_{n=n_{0}+1}^{\infty} n^{r-1} h(n) P\left\{\left|X_{1}\right|>\varepsilon n^{1 / q} \delta\right\} \\
& \leq E\left(\left|\frac{X_{1}}{\varepsilon \delta}\right|^{r q} h\left(\left|\frac{X_{1}}{\varepsilon \delta}\right|^{q}\right)\right) .
\end{aligned}
$$

The last inequality holds by Lemma 3.2. From (2.1) it follows that

$$
E\left(\left|\frac{X_{1}}{\varepsilon \delta}\right|^{r q} h\left(\left|\frac{X_{1}}{\varepsilon \delta}\right|^{q}\right)\right) \leq \text { const. } E\left(\left|X_{1}\right|^{r q} h\left(\left|X_{1}\right|^{q}\right)\right) .
$$

Condition (2.4) and inequalities (2.14)-(2.17) imply (2.5) for $0<r q<1$.

Now assume that $r q \geq 1$. First we consider the case $1 \leq r q \leq 2$. Note that

$$
P\left\{\max _{1 \leq k \leq n}\left|S_{k}\right|>\varepsilon n^{1 / q}\right\} \leq P\left\{\max _{1 \leq k \leq n}\left|S_{k}-E S_{n}^{\prime}\right|>\varepsilon n^{1 / q}-\max _{1 \leq k \leq n}\left|E S_{k}^{\prime}\right|\right\}
$$

By Lemma 3.4, we have $\max _{1 \leq k \leq n} E\left|S_{k}^{\prime}\right| n^{-1 / q} \rightarrow 0$ as $n \rightarrow \infty$, and hence

$$
P\left\{\max _{1 \leq k \leq n}\left|S_{k}\right|>\varepsilon n^{1 / q}\right\} \leq P\left\{\max _{1 \leq k \leq n}\left|S_{k}-E S_{n}^{\prime}\right|>\frac{\varepsilon n^{1 / q}}{2}\right\}
$$

for all $n \in \mathbb{N}$ greater than some $n_{0}^{\prime}$. The probability on the right-hand side can be estimated as follows:

$$
\begin{aligned}
P\left\{\max _{1 \leq k \leq n}\left|S_{k}-E S_{k}^{\prime}\right|>\frac{\varepsilon n^{1 / q}}{2}\right\}= & P\left\{\left\{\max _{1 \leq k \leq n}\left|S_{k}^{\prime}-E S_{k}^{\prime}\right|>\frac{\varepsilon n^{1 / q}}{2}\right\} \cap \bigcap_{k=1}^{n}\left\{X_{k}=Y_{k}\right\}\right\} \\
& +P\left\{\left\{\max _{1 \leq k \leq n}\left|S_{k}-E S_{k}^{\prime}\right|>\frac{\varepsilon n^{1 / q}}{2}\right\} \cap\left(\bigcup_{k=1}^{n}\left\{X_{k} \neq Y_{k}\right\}\right)\right\} \\
\leq & P\left\{\max _{1 \leq k \leq n}\left|S_{k}^{\prime}-E S_{k}^{\prime}\right|>\frac{\varepsilon n^{1 / q}}{2}\right\}+P\left\{\max _{1 \leq k \leq n}\left|X_{k}\right|>\varepsilon n^{1 / q} \delta\right\} .
\end{aligned}
$$


In order to apply Lemma 3.1, put $\gamma=r q, x=\varepsilon n^{1 / q} / 2, c=\varepsilon n^{1 / q}(r-1) /(4(r+l))$. From (2.13) it follows that the inequality $E\left|Y_{1}-E Y_{1}\right|^{\gamma}<2 E\left|X_{1}-E X_{1}\right|^{\gamma}$ holds for all $n \in \mathbb{N}$ greater than some $n_{0}^{\prime \prime} \geq n_{0}^{\prime}$. By inequality (3.2) we get, for $n>n_{0}^{\prime \prime}$,

$$
\begin{aligned}
P\left\{\max _{1 \leq k \leq n}\left|S_{k}^{\prime}-E S_{k}^{\prime}\right|>\frac{\varepsilon n^{1 / q}}{2}\right\} \\
\quad \leq 4 \exp \left\{\frac{x}{2^{r-1} c}-\frac{x}{2 c} \ln \left(\frac{x c^{\gamma-1}}{n E\left|Y_{1}-E Y_{1}\right|^{r}}+1\right)\right\} \\
\quad \leq 4 \exp \left\{\frac{2(r+l)}{r-1}-\frac{r+l}{r-1} \ln \left(\frac{\varepsilon^{r q}(r-1)^{r q-1}}{2 E\left|X_{1}-E X_{1}\right|^{r q}(4(r+l))^{r q-1}} n^{r-1}+1\right)\right\} \\
\quad \leq 4 \exp \left\{\frac{2(r+l)}{r-1}\right\}\left(\frac{\varepsilon^{r q}(r-1)^{r q-1}}{2 E\left|X_{1}-E X_{1}\right|^{r q}(4(r+l))^{r q-1}}\right)^{-(r+l) /(r-1)} n^{-(r+l)}=C_{1} n^{-(r+l)} .
\end{aligned}
$$

These inequalities and (2.3) imply

$$
\sum_{n=n_{0}^{\prime \prime}+1}^{\infty} n^{r-2} h(n) P\left\{\max _{1 \leq k \leq n}\left|S_{k}^{\prime}-E S_{k}^{\prime}\right|>\frac{\varepsilon n^{1 / q}}{2}\right\} \leq \Delta C_{1} \sum_{n=n_{0}^{\prime \prime}+1}^{\infty} n^{r+l-2} n^{-(r+l)}<\infty .
$$

From this inequality and (2.17) with $n_{0}^{\prime \prime}$ instead of $n_{0},(2.20)$ and (2.21), it follows that (2.5) holds for $1 \leq r q \leq 2$. The case $r q>2$ can be considered in the same way. In order to apply Lemma 3.1, put $\gamma=2, x=\varepsilon n^{1 / q} / 2, c=\varepsilon n^{1 / q}(2-q) /(4 q(r+l))$. From (2.13) it follows that the inequality $E\left|Y_{1}-E Y_{1}\right|^{2}<2 E\left|X_{1}-E X_{1}\right|^{2}$ holds for all $n \in \mathbb{N}$ greater than some $n_{0}^{\prime \prime \prime} \geq n_{0}^{\prime}$. By inequality (3.2), we get, for $n>n_{0}^{\prime \prime \prime}$,

$$
\begin{aligned}
P\left\{\max _{1 \leq k \leq n}\left|S_{k}^{\prime}-E S_{k}^{\prime}\right|>\frac{\varepsilon n^{1 / q}}{2}\right\} \\
\quad \leq 4 \exp \left\{\frac{x}{2 c}-\frac{x}{2 c} \ln \left(\frac{\mathrm{xc}}{n E\left|Y_{1}-E Y_{1}\right|^{2}}+1\right)\right\} \\
\quad \leq 4 \exp \left\{\frac{q(r+l)}{2-q}-\frac{q(r+l)}{2-q} \ln \left(\frac{\varepsilon^{2}(2-q)}{16 E\left|X_{1}-E X_{1}\right|^{2} q(r+l)} n^{2 / q-1}+1\right)\right\} \\
\quad \leq 4 \exp \left\{\frac{q(r+l)}{2-q}\right\}\left(\frac{\varepsilon^{2}(2-q)}{16 E\left|X_{1}-E X_{1}\right|^{2} q(r+l)}\right)^{-q(r+l) /(2-q)} n^{-(r+l)}=C_{2} n^{-(r+l)}
\end{aligned}
$$

These inequalities and (2.3) imply

$$
\sum_{n=n_{0}^{\prime \prime \prime}+1}^{\infty} n^{r-2} h(n) P\left\{\max _{1 \leq k \leq n}\left|S_{k}^{\prime}-E S_{k}^{\prime}\right|>\frac{\varepsilon n^{1 / q}}{2}\right\} \leq \Delta C_{2} \sum_{n=n_{0}^{\prime \prime \prime}+1}^{\infty} n^{r+l-2} n^{-(r+l)}<\infty .
$$


From this inequality and (2.17) with $n_{0}^{\prime \prime \prime}$ instead of $n_{0},(2.20)$ and (2.21), it follows that (2.5) holds for $r q>2$.

Prove that $(2.5) \Rightarrow(2.6)$. Note that

$$
\begin{aligned}
\sum_{n=3}^{\infty} n^{r-2} h(n) P\left\{\sup _{k \geq n}\left|k^{-1 / q} S_{k}\right|>\varepsilon\right\} & =\sum_{j=1}^{\infty} \sum_{n=2^{j+1}}^{2^{j+1}} n^{r-2} h(n) P\left\{\sup _{k \geq n}\left|k^{-1 / q} S_{k}\right|>\varepsilon\right\} \\
& \leq \sum_{j=1}^{\infty} 2^{(j+1)(r-1)} h\left(2^{j+1}\right) P\left\{\sup _{k \geq 2^{j}}\left|k^{-1 / q} S_{k}\right|>\varepsilon\right\} \\
& \leq \sum_{j=1}^{\infty} 2^{(j+1)(r-1)} h\left(2^{j+1}\right) \sum_{i=j}^{\infty} P\left\{\max _{2^{i} \leq k \leq 2^{i+1}}\left|k^{-1 / q} S_{k}\right|>\varepsilon\right\} \\
& =\sum_{i=1}^{\infty} \sum_{j=1}^{i} 2^{(j+1)(r-1)} h\left(2^{j+1}\right) P\left\{\max _{2^{i} \leq k \leq 2^{i+1}}\left|k^{-1 / q} S_{k}\right|>\varepsilon\right\} \\
& \leq \frac{2^{r-1}}{2^{r-1}-1} \sum_{i=1}^{\infty} 2^{(i+1)(r-1)} h\left(2^{i+1}\right) P\left\{\max _{2^{i} \leq k \leq 2^{i+1}}\left|S_{k}\right|>\varepsilon 2^{\mathrm{i} / q}\right\} .
\end{aligned}
$$

The last series can be estimated as follows:

$$
\begin{aligned}
& \sum_{i=1}^{\infty} 2^{(i+1)(r-1)} h\left(2^{i+1}\right) P\left\{\max _{2^{i} \leq k \leq 2^{i+1}}\left|S_{k}\right|>\varepsilon 2^{\mathrm{i} / q}\right\} \\
& \quad \leq 2^{5 r-3} h\left(2^{5}\right)+\max \left\{1,2^{2-r}\right\} \sum_{i=1}^{\infty} \sum_{n=2^{i+1}+1}^{2^{i+2}} n^{r-2} h(n) P\left\{\max _{1 \leq k \leq n}\left|S_{k}\right|>\varepsilon 2^{-2 / q} n^{1 / q}\right\} \\
& \quad \leq 2^{5 r-3} h\left(2^{5}\right)+\max \left\{1,2^{2-r}\right\} \sum_{n=1}^{\infty} n^{r-2} h(n) P\left\{\max _{1 \leq k \leq n}\left|S_{k}\right|>\varepsilon 2^{-2 / q} n^{1 / q}\right\}
\end{aligned}
$$

Condition (2.5) and these inequalities imply (2.6).

Prove that $(2.6) \Rightarrow(2.4)$. The sequence $\left\{P\left\{\sup _{k \geq n}\left|k^{-1 / q} S_{k}\right|>\varepsilon\right\}\right\}_{n \geq 1}$ decreases to zero for any $\varepsilon>0$. Indeed, if the sequence converges to a number $a>0$, then

$$
\infty=a \sum_{n=1}^{\infty} n^{r-2} h(n) \leq \sum_{n=1}^{\infty} n^{r-2} h(n) P\left\{\sup _{k \geq n}\left|k^{-1 / q} S_{k}\right|>\varepsilon\right\}<\infty .
$$

Note that $P\left\{\max _{n \leq k \leq 2 n}\left|S_{k}\right|>\varepsilon(2 n)^{1 / q}\right\} \leq P\left\{\sup _{k \geq n}\left|k^{-1 / q} S_{k}\right|>\varepsilon\right\} \rightarrow 0$ as $n \rightarrow \infty$. 
Journal of Probability and Statistics

With the help of (1.2), we obtain

$$
\begin{aligned}
P\left\{\max _{n<k \leq 2 n} X_{k}>n^{1 / q}\right\} & =1-P\left\{\max _{n<k \leq 2 n} X_{k} \leq n^{1 / q}\right\} \\
& \geq 1-\prod_{k=n+1}^{2 n} P\left\{X_{k} \leq n^{1 / q}\right\}=1-\left(1-P\left\{X_{1}>n^{1 / q}\right\}\right)^{n}, \\
P\left\{\max _{n<k \leq 2 n}\left(-X_{k}\right)>n^{1 / q}\right\} & =1-P\left\{\max _{n<k \leq 2 n}\left(-X_{k}\right) \leq n^{1 / q}\right\} \\
& \geq 1-\prod_{k=n+1}^{2 n} P\left\{X_{k} \geq-n^{1 / q}\right\}=1-\left(1-P\left\{-X_{1}>n^{1 / q}\right\}\right)^{n} .
\end{aligned}
$$

Denote $a_{n}^{+}=P\left\{X_{1}>n^{1 / q}\right\}$ and $a_{n}^{-}=P\left\{-X_{1}>n^{1 / q}\right\}$. Note that $P\left\{\left|X_{1}\right|>n^{1 / q}\right\}=a_{n}^{+}+a_{n}^{-}$and $1-\left(1-a_{n}^{ \pm}\right)^{n} \leq P\left\{\max _{n<k \leq 2 n}\left|X_{k}\right|>n^{1 / q}\right\} \leq P\left\{\max _{n \leq k \leq 2 n}\left|S_{k}\right|>n^{1 / q} / 2\right\}$. Since $P\left\{\max _{n \leq k \leq 2 n}\left|S_{k}\right|>\right.$ $\left.n^{1 / q} / 2\right\} \rightarrow 0$ and $a_{n}^{ \pm} \leq P\left\{\left|X_{1}\right|>n^{1 / q}\right\} \rightarrow 0$ as $n \rightarrow \infty$, then $n \ln \left(1-a_{n}^{ \pm}\right)>-\ln 2$ and $0 \leq a_{n}^{+}+a_{n}^{-}<1$ for all $n \in \mathbb{N}$ greater than some $n_{0}$. By the inequalities $-\ln (1-x) \geq x$ for $x \in[0,1)$ and $1-e^{x} \geq e^{x}|x|$ for $x \leq 0$, we obtain

$$
n a_{n}^{ \pm} \leq-n \ln \left(1-a_{n}^{ \pm}\right) \leq 2\left(1-e^{n \ln \left(1-a_{n}^{ \pm}\right)}\right)=2\left(1-\left(1-a_{n}^{ \pm}\right)^{n}\right) \leq 2 P\left\{\max _{1 \leq k \leq n}\left|X_{k}\right|>n^{1 / q}\right\}
$$

for all $n>n_{0}$, and

$$
\begin{aligned}
\sum_{n=n_{0}+1}^{\infty} n^{r-1} h(n) P\left\{\left|X_{1}\right|>n^{1 / q}\right\} & =\sum_{n=n_{0}+1} n^{r-1} h(n)\left(a_{n}^{+}+a_{n}^{-}\right) \\
& \leq 4 \sum_{n=n_{0}+1}^{\infty} n^{r-2} h(n) P\left\{\max _{n<k \leq 2 n}\left|X_{k}\right|>n^{1 / q}\right\} \\
& \leq 4 \sum_{n=1}^{\infty} n^{r-2} h(n) P\left\{\max _{n \leq k \leq 2 n}\left|S_{k}\right|>\frac{n^{1 / q}}{2}\right\} \\
& \leq 4 \sum_{n=1}^{\infty} n^{r-2} h(n) P\left\{\max _{n \leq k \leq 2 n}\left|k^{-1 / q} S_{k}\right|>\frac{2^{-1 / q}}{2}\right\} \\
& \leq 4 \sum_{n=1}^{\infty} n^{r-2} h(n) P\left\{\sup _{k \geq n}\left|k^{-1 / q} S_{k}\right|>\frac{2^{-1 / q}}{2}\right\}<\infty .
\end{aligned}
$$

It follows by Lemma 3.3 that $E\left(\left|X_{1}\right|^{r q} h\left(\left|X_{1}\right|^{q}\right)\right)<\infty$.

Now we will prove that $a=E X_{1}=0$ provided that $1 \leq q<2$. Assume that $a \neq 0$. Since $n|a| \leq\left|S_{n}-n a\right|+\left|S_{n}\right|$, then

$$
1=P\left\{\left|S_{n}-n a\right|+\left|S_{n}\right|>\frac{|a| n^{1 / q}}{2}\right\} \leq P\left\{\left|S_{n}-n a\right|>\frac{|a| n^{1 / q}}{4}\right\}+P\left\{\left|S_{n}\right|>\frac{|a| n^{1 / q}}{4}\right\},
$$


and hence

$$
\infty=\sum_{n=1}^{\infty} n^{r-2} h(n) \leq \sum_{n=1}^{\infty} n^{r-2} h(n) P\left\{\left|S_{n}-n a\right|>\frac{|a| n^{1 / q}}{4}\right\}+\sum_{n=1}^{\infty} n^{r-2} h(n) P\left\{\left|S_{n}\right|>\frac{|a| n^{1 / q}}{4}\right\} .
$$

But this contradicts the convergence of the series on the right-hand side of the inequality. The equality $E X_{1}=0$ is proved.

Proof of Theorem 2.5. Both theorems overlap. We need to consider only the case $r=1$. Prove that $(2.8) \Rightarrow(2.9)$. Define random variables $Z_{n}=-\varepsilon n^{1 / q} I_{\left(-\infty,-\varepsilon n^{1 / q)}\right.}\left(X_{n}\right)+X_{n} I_{\left[-\varepsilon n^{1 /,}, \varepsilon n^{1 / q}\right]}\left(X_{n}\right)+$ $\varepsilon n^{1 / q} I_{\left(\varepsilon n^{1 / q, \infty}\right)}\left(X_{n}\right), n \in \mathbb{N}$. By Theorem $A$ the random variables $Z_{n}, n \in \mathbb{N}$, as well as $Z_{n}-E Z_{n}, n \in \mathbb{N}$, are negatively associated. Denote $S_{k}^{\prime \prime}=\left(Z_{1}-E Z_{1}\right)+\cdots+\left(Z_{k}-E Z_{k}\right)$. By Theorem $\mathrm{B}$ there exist independent random variables $X_{1}^{*}, \ldots, X_{n}^{*}$ such that the random variables $Z_{k}-E Z_{k}$ and $X_{k}^{*}$ are identically distributed for all $k=1, \ldots, n$, and $E\left(\max _{1 \leq k \leq n}\left|S_{k}^{\prime \prime}\right|^{2}\right) \leq$ $2 E\left(X_{1}^{*}+\cdots+X_{n}^{*}\right)^{2}$. Similarly to $(2.21)$, we can prove that

$$
P\left\{\max _{1 \leq k \leq n}\left|S_{k}\right|>\varepsilon n^{1 / q}\right\} \leq P\left\{\max _{1 \leq k \leq n}\left|S_{k}^{\prime \prime}-E S_{k}^{\prime \prime}\right|>\frac{\varepsilon n^{1 / q}}{2}\right\}+P\left\{\max _{1 \leq k \leq n}\left|X_{k}\right|>\varepsilon n^{1 / q}\right\}
$$

for all $n \in \mathbb{N}$ grater than some $n_{0}$. In the same way as (2.17), one can prove that

$$
\begin{aligned}
\sum_{n=n_{0}+1}^{\infty} \frac{h(n)}{n} P\left\{\max _{1 \leq k \leq n}\left|X_{k}\right|>\varepsilon n^{1 / q}\right\} & \leq \sum_{n=n_{0}+1}^{\infty} h(n) P\left\{\left|X_{1}\right|>\varepsilon n^{1 / q}\right\} \\
& \leq E\left(\left|\frac{X_{1}}{\varepsilon}\right|^{q} h\left(\left|\frac{X_{1}}{\varepsilon}\right|^{q}\right)\right)<\infty .
\end{aligned}
$$

With the help of the Markov inequality, we obtain

$$
\begin{aligned}
P\left\{\max _{1 \leq k \leq n}\left|S_{k}^{\prime \prime}-E S_{k}^{\prime \prime}\right|>\frac{\varepsilon n^{1 / q}}{2}\right\} & \leq 4 \varepsilon^{-2} n^{-2 / q} E\left(\max _{1 \leq k \leq n}\left|S_{k}^{\prime \prime}-E S_{k}^{\prime \prime}\right|^{2}\right) \\
& \leq 8 \varepsilon^{-2} n^{-2 / q} E\left(X_{1}^{*}+\cdots+X_{n}^{*}\right)^{2} \\
& =8 \varepsilon^{-2} n^{1-2 / q} E\left|Z_{1}-Z Y_{1}\right|^{2} .
\end{aligned}
$$

From (2.34) and (2.35), it follows that (2.9) holds if the series $\sum_{n=1}^{\infty} h(n) n^{-2 / q} E\left|Z_{1}-E Z_{1}\right|^{2}$ converges. This series can be estimated as follows:

$$
\begin{aligned}
\sum_{n=1}^{\infty} \frac{h(n)}{n^{2 / q}} E\left|Z_{1}-E Z_{1}\right|^{2} & \leq \sum_{n=1}^{\infty} \frac{h(n)}{n^{2 / q}} E\left|Z_{1}\right|^{2} \\
& =\sum_{n=1}^{\infty} \frac{h(n)}{n^{2 / q}} E\left(X_{1}^{2} I_{\left\{\left|X_{1}\right| \leq \varepsilon n^{1 / q}\right\}}\right)+\varepsilon^{2} \sum_{k=1}^{\infty} h(n) P\left\{\left|X_{1}\right|>\varepsilon n^{1 / q}\right\} .
\end{aligned}
$$


Journal of Probability and Statistics

Rewrite the first summand on the right-hand side in the following way:

$$
\begin{aligned}
\sum_{n=1}^{\infty} \frac{h(n)}{n^{2 / q}} E\left(\left|X_{1}\right|^{2} I_{\left\{\left|X_{1}\right| \leq \varepsilon n^{1 / q}\right\}}\right) & =\sum_{n=1}^{\infty} \frac{h(n)}{n^{2 / q}} \sum_{k=1}^{n} E\left(X_{1}^{2} I_{\left\{\varepsilon(k-1)^{1 / q}<\left|X_{1}\right| \leq \varepsilon k^{1 / q}\right\}}\right) \\
& =\sum_{k=1}^{\infty} \sum_{n=k}^{\infty} \frac{h(n)}{n^{2 / q}} E\left(X_{1}^{2} I_{\left\{\varepsilon(k-1)^{1 / q}<\left|X_{1}\right| \leq \varepsilon k^{1 / q}\right\}}\right) .
\end{aligned}
$$

From (2.1) and (2.2), it follows that there exist numbers $k_{0} \in \mathbb{N}, k_{0}>2$, and $C>0$ such that

$$
h(k) \leq C h\left(\frac{k}{2}\right), \quad \int_{k}^{\infty} h(x) x^{-2 / q} d x \leq C h(k) k^{1-2 / q}, \quad \forall k \geq k_{0} .
$$

For any $k \in \mathbb{N}$, we have

$$
\sum_{n=k}^{\infty} \frac{h(n)}{n^{2 / q}} \leq 2^{2 / q} \sum_{n=k}^{\infty} \int_{n}^{n+1} \frac{h(x)}{x^{2 / q}} d x=2^{2 / q} \int_{k}^{\infty} \frac{h(x)}{x^{2 / q}} d x<\infty
$$

If $k>k_{0}$, then

$$
\sum_{n=k}^{\infty} \frac{h(n)}{n^{2 / q}} \leq 2^{2 / q} \int_{k}^{\infty} \frac{h(x)}{x^{2 / q}} d x \leq 2^{2 / q} C h(k) k^{1-2 / q} \leq 2^{2 / q} C^{2} h(k-1)(k-1)^{1-2 / q} .
$$

With the help of these estimates, we get

$$
\begin{aligned}
\sum_{n=1}^{\infty} \frac{h(n)}{n^{2 / q}} E\left(\left|X_{1}\right|^{2} I_{\left\{\left|X_{1}\right| \leq \varepsilon n^{1 / q}\right\}}\right) \leq 2^{2 / q} \sum_{k=1}^{k_{0}} \int_{k}^{\infty} \frac{h(x)}{x^{2 / q}} d x \\
+2^{2 / q} C^{2} \sum_{k=k_{0}+1}^{\infty} \frac{h(k-1)}{(k-1)^{2 / q-1}} E\left(X_{1}^{2} I_{\left\{\varepsilon(k-1)^{1 / q}<\left|X_{1}\right| \leq \varepsilon k^{1 / q}\right\}}\right) .
\end{aligned}
$$

The last series can be estimated as follows:

$$
\begin{aligned}
& \sum_{k=k_{0}+1}^{\infty} \frac{h(k-1)}{(k-1)^{2 / q-1}} E\left(X_{1}^{2} I_{\left\{\varepsilon(k-1)^{1 / q}<\left|X_{1}\right| \leq \varepsilon k^{1 / q}\right\}}\right) \\
& \leq \varepsilon^{2} 2^{2 / q-1} \sum_{k=k_{0}+1}^{\infty} E\left(\left|\varepsilon^{-1} X\right|^{q} h\left(\left|\varepsilon^{-1} X_{1}\right|^{q}\right) I_{\left\{\varepsilon(k-1)^{1 / q}<\left|X_{1}\right| \leq \varepsilon k^{1 / q}\right\}}\right) \\
& \leq \varepsilon^{2} 2^{2 / q-1} E\left(\left|\varepsilon^{-1} X_{1}\right|^{q} h\left(\left|\varepsilon^{-1} X_{1}\right|^{q}\right)\right)<\infty .
\end{aligned}
$$

As a result we get that $\sum_{n=1}^{\infty} h(n) n^{-2 / q} E\left(\left|X_{1}\right|^{2} I_{\left\{\left|X_{1}\right| \leq \varepsilon n^{1 / q\}}\right.}\right)<\infty$. Taking account of (2.35) and (2.37), we see that $\sum_{n=1}^{\infty} h(n) n^{-2 / q} E\left|Z_{1}-E Z_{1}\right|^{2}<\infty$. 
Prove that $(2.9) \Rightarrow(2.8)$ for $r=1$. Note that

$$
\begin{aligned}
\sum_{n=1}^{\infty} h\left(2^{n}\right) P\left\{\max _{2^{n}<k \leq 2^{n+1}}\left|X_{k}\right|>2^{n / q}\right\} & \leq \sum_{n=1}^{\infty} h\left(2^{n}\right) P\left\{\max _{2^{n} \leq k \leq 2^{n+1}}\left|S_{k}\right|>\frac{2^{n / q}}{2}\right\} \\
& \leq 2 \sum_{n=1}^{\infty} \sum_{j=2^{n+1}}^{2^{n+2}-1} \frac{h(j)}{j} P\left\{\max _{2^{n} \leq k \leq j}\left|S_{k}\right|>\frac{2^{-2 / q} j^{1 / q}}{2}\right\} \\
& \leq 2 \sum_{n=1}^{\infty} \frac{h(n)}{n} P\left\{\max _{1 \leq k \leq n}\left|S_{k}\right|>\frac{2^{-2 / q} n^{1 / q}}{2}\right\}<\infty .
\end{aligned}
$$

Denote $b_{n}^{+}=P\left\{X_{1}>2^{n / q}\right\}$ and $b_{n}^{-}=P\left\{-X_{1}>2^{n / q}\right\}$. Note that $P\left\{\left|X_{1}\right|>2^{n / q}\right\}=b_{n}^{+}+b_{n}^{-}$. With the help of (1.2), one can prove the inequality $1-\left(1-b_{n}^{ \pm}\right)^{2^{n}} \leq P\left\{\max _{2^{n+1}<k \leq 2^{n+2}}\left|X_{k}\right|>2^{n / q}\right\}$. Since $P\left\{\max _{2^{n}<k \leq 2^{n+1}}\left|X_{k}\right|>2^{n / q}\right\} \rightarrow 0$ and $b_{n}^{ \pm} \leq P\left\{\left|X_{1}\right|>2^{n / q}\right\} \rightarrow 0$ as $n \rightarrow \infty$, then $2^{n} \ln \left(1-b_{n}^{ \pm}\right)>$ $-\ln 2$ and $0 \leq b_{n}^{+}+b_{n}^{-}<1$ for all $n \in \mathbb{N}$ greater than some $n_{0}$. By the inequalities $-\ln (1-x) \geq x$ for $x \in[0,1)$ and $1-e^{x} \geq e^{x}|x|$ for $x \leq 0$, we obtain

$$
\begin{aligned}
2^{n} b_{n}^{ \pm} & \leq-2^{n} \ln \left(1-b_{n}^{ \pm}\right) \leq 2\left(1-e^{2^{n} \ln \left(1-b_{n}^{ \pm}\right)}\right) \\
& =2\left(1-\left(1-b_{n}^{ \pm}\right)^{2^{n}}\right) \\
& \leq 2 P\left\{\max _{2^{n+1}<k \leq 2^{n+2}}\left|X_{k}\right|>2^{n / q}\right\}, \\
\sum_{n=1}^{\infty} h\left(2^{n}\right)\left(2^{n+1}-2^{n}\right) P\left\{\left|X_{1}\right|>2^{n / q}\right\} & =\sum_{n=1}^{\infty} h\left(2^{n}\right) 2^{n}\left(b_{n}^{+}+b_{n}^{-}\right) \\
& \leq 4 \sum_{n=1}^{\infty} h\left(2^{n}\right) P\left\{\max _{2^{n}<k \leq 2^{n+1}}\left|X_{k}\right|>2^{n / q}\right\}<\infty .
\end{aligned}
$$

By Lemma 3.3, we have that $E\left(\left|X_{1}\right|^{q} h\left(\left|X_{1}\right|^{q}\right)\right)<\infty$. In the same way as in the proof of the previous theorem, one can prove that $E X_{1}=0$ if $q \geq 1$.

\section{Auxiliary Results}

Let $X_{1}, \ldots, X_{n}$ be random variables. Denote $S_{k}=X_{1}+\cdots+X_{k}$ for $k=1, \ldots, n$ and $A_{n, r}=$ $E\left|X_{1}\right|^{\gamma}+\cdots+E\left|X_{n}\right|^{\gamma}, B_{n, \gamma}=E\left|X_{1}-E X_{1}\right|^{\gamma}+\cdots+E\left|X_{n}-E X_{n}\right|^{\gamma}$ for some $\gamma \in(0,2], \cosh x=$ $\left(e^{x}+e^{-x}\right) / 2, x \in \mathbb{R}=(-\infty, \infty)$. 
Journal of Probability and Statistics

Lemma 3.1. If negatively associated random variables $X_{1}, \ldots, X_{n}$ are bounded by a constant $c>0$, then

$$
\begin{gathered}
P\left\{\max _{1 \leq k \leq n}\left|S_{k}\right|>x\right\} \leq 2 \exp \left\{\frac{x}{c}-\frac{x}{c} \ln \left(\frac{x c^{\gamma-1}}{A_{n, \gamma}}+1\right)\right\}, \quad 0<\gamma \leq 1, \\
P\left\{\max _{1 \leq k \leq n}\left|S_{k}-E S_{k}\right|>x\right\} \leq 4 \exp \left\{\frac{x}{2^{\gamma-1} c}-\frac{x}{2 c} \ln \left(\frac{x c^{\gamma-1}}{B_{n, \gamma}}+1\right)\right\}, \quad 1 \leq r \leq 2,
\end{gathered}
$$

for any number $x>0$.

Proof. Prove Inequality (3.1). It is easily verified that $\left\{\max _{1 \leq k \leq n}\left|S_{k}\right|>x\right\} \subseteq\left\{\sum_{k=1}^{n} X_{k}^{+}>x\right\} \cup$ $\left\{\sum_{k=1}^{n} X_{k}^{-}>x\right\}$ where $X_{k}^{+}=\max \left\{0, X_{k}\right\}$ and $X_{k}^{-}=\max \left\{0,-X_{k}\right\}$. With the help of Markov inequality, we get

$$
\begin{aligned}
P\left\{\max _{1 \leq k \leq n}\left|S_{k}\right|>x\right\} & \leq P\left\{\sum_{k=1}^{n} X_{k}^{+} \mid>x\right\}+P\left\{\sum_{k=1}^{n} X_{k}^{-}>x\right\} \\
& \leq e^{-h x} E \exp \left\{h \sum_{k=1}^{n} X_{k}^{+}\right\}+e^{-h x} E \exp \left\{h \sum_{k=1}^{n} X_{k}^{-}\right\}
\end{aligned}
$$

for any $h>0$. By Theorem A, random variables $X_{k}^{+}, k=1, \ldots, n$, as well as $X_{k}^{-}, k=1, \ldots, n$, are negatively associated. By Theorem $\mathrm{B}$, the inequality

$$
E \exp \left\{h\left(X_{1}^{ \pm}+\cdots+X_{n}^{ \pm}\right)\right\} \leq E \exp \left\{h\left(\left(X_{1}^{*}\right)^{ \pm}+\cdots+\left(X_{n}^{*}\right)^{ \pm}\right)\right\}
$$

holds where random variables $X_{1}^{*}, \ldots, X_{n}^{*}$ are independent, and for any $k=1, \ldots, n$, random variables $X_{k}$ and $X_{k}^{*}$ are identically distributed. It follows that

$$
\begin{aligned}
P\left\{\max _{1 \leq k \leq n}\left|S_{k}\right|>x\right\} & \leq e^{-h x} \prod_{k=1}^{n} E e^{h\left(X_{k}^{*}\right)^{+}}+e^{-h x} \prod_{k=1}^{n} E e^{h\left(X_{k}^{*}\right)^{-}} \\
& =e^{-h x} \prod_{k=1}^{n} E e^{h X_{k}^{+}}+e^{-h x} \prod_{k=1}^{n} E e^{h X_{k}^{-}} \leq 2 e^{-h x} \prod_{k=1}^{n} E e^{h\left|X_{k}\right|} .
\end{aligned}
$$


Further we can proceed as in Prokhorov [12], Fuk and Nagaev [13], and Kruglov [14]. Assume that $0<\gamma \leq 1$. The function $\left(e^{h x}-1\right) / x^{r}, 0 \leq x \leq c$, increases and hence $\left(e^{h x}-1\right) / x^{\gamma} \leq\left(e^{h c}-1\right) / c^{\gamma}$. With the help of this inequality, we obtain

$$
\begin{aligned}
\prod_{k=1}^{n} E e^{h\left|X_{k}\right|} & =\prod_{k=1}^{n} E\left(1+\left|X_{k}\right|^{-\gamma}\left(e^{h\left|X_{k}\right|}-1\right)\left|X_{k}\right|^{\gamma}\right) \\
& \leq \prod_{k=1}^{n}\left(1+c^{-\gamma}\left(e^{h c}-1\right) E\left|X_{k}\right|^{\gamma}\right) \\
& =\exp \left\{c^{-\gamma}\left(e^{h c}-1\right) A_{n, \gamma}\right\} .
\end{aligned}
$$

From this inequality and from (3.5), it follows

$$
P\left\{\max _{1 \leq k \leq n}\left|S_{k}\right|>x\right\} \leq 2 \exp \left\{-h x+c^{-r}\left(e^{h c}-1\right) A_{n, r}\right\} .
$$

Put $h=c^{-1} \ln \left(x c^{\gamma-1} / A_{n, \gamma}+1\right)$. As a result we obtain (3.1).

Now we assume that $1 \leq r \leq 2$. By the Markov inequality we get

$$
P\left\{\max _{1 \leq k \leq n}\left|S_{k}-E S_{\mathrm{k}}\right|>x\right\} \leq \frac{E \cosh \left(h \max _{1 \leq k \leq n}\left|S_{k}-E S_{k}\right|\right)}{\cosh (h x)}
$$

for any $h>0$. By Theorem B, the inequality

$$
E\left(\max _{1 \leq k \leq n}\left|S_{k}-E S_{k}\right|\right)^{r} \leq 2 E\left|\left(X_{1}^{*}-E X_{1}^{*}\right)+\cdots+\left(X_{n}^{*}-E X_{n}^{*}\right)\right|^{r}
$$

holds for any $r>1$. Denote $S_{n}^{*}=X_{1}^{*}+\cdots+X_{n}^{*}$. Note that $\cosh (|x|)=\cosh (x)$ for any $x \in \mathbb{R}$. With these remarks, we obtain

$$
\begin{aligned}
E \cosh \left(h \max _{1 \leq k \leq n}\left|S_{k}-E S_{k}\right|\right) & =1+\sum_{r=1}^{\infty} \frac{E\left(h \max _{1 \leq k \leq n}\left|S_{\mathrm{k}}-E S_{k}\right|^{2 r}\right)}{(2 r) !} \\
& \leq 1+2 \sum_{r=1}^{\infty} \frac{E\left|h\left(S_{n}^{*}-E S_{n}^{*}\right)\right|^{2 r}}{(2 r) !} \\
& \leq 2 E \cosh \left(h\left|S_{n}^{*}-E S_{n}^{*}\right|\right)=2 E \cosh \left(h\left(S_{n}^{*}-E S_{n}^{*}\right)\right),
\end{aligned}
$$

and hence

$$
\begin{aligned}
P\left\{\max _{1 \leq k \leq n}\left|S_{\mathrm{k}}-E S_{k}\right|>x\right\} & \leq 2 \frac{E \cosh \left(h\left(S_{n}^{*}-E S_{n}^{*}\right)\right)}{\cosh (h x)} \\
& \leq 2 e^{-h x}\left(E e^{h\left(S_{n}^{*}-E S_{n}^{*}\right)}+E e^{-h\left(S_{n}^{*}-E S_{n}^{*}\right)}\right) .
\end{aligned}
$$


By the inequalities $\alpha \leq e^{\alpha-1}$ and $e^{\alpha}-1-\alpha \leq \cosh \alpha-1$ for $\alpha \in \mathbb{R}$, we obtain

$$
\begin{aligned}
E e^{h\left(S_{n}^{*}-E S_{n}^{*}\right)}=\prod_{k=1}^{n} E e^{h\left(X_{k}^{*}-E X_{k}^{*}\right)} & \leq \prod_{k=1}^{n} \exp \left\{E\left(e^{h\left(X_{k}^{*}-E X_{k}^{*}\right)}-1\right)\right\} \\
& =\exp \left\{\sum_{k=1}^{n} E\left(e^{h\left(X_{k}^{*}-E X_{k}^{*}\right)}-1-h\left(X_{k}^{*}-E X_{k}^{*}\right)\right)\right\} \\
& \leq \exp \left\{2 \sum_{k=1}^{n} E\left(\cosh \left(h\left(X_{k}^{*}-E X_{k}^{*}\right)\right)-1\right)\right\} .
\end{aligned}
$$

Put $f(\alpha)=(\cosh \alpha-1)|\alpha|^{-\gamma}$ for $\alpha \neq 0$ and $f(0)=1 / 2$ if $\gamma=2$, and $f(0)=0$ if $1 \leq \gamma<2$. It can be easily verified that the function $f$ is continuous, even, and increases on $(0, \infty)$. Note that $\left|X_{k}^{*}-E X_{k}^{*}\right| \leq 2 c$ for all $k=1, \ldots, n$. With these remarks, we obtain

$$
\begin{aligned}
\cosh \left(h\left(X_{k}^{*}-E X_{k}^{*}\right)\right)-1 & =\left(\cosh \left(h\left(X_{k}^{*}-E X_{k}^{*}\right)\right)-1\right)\left|h\left(X_{k}^{*}-E X_{k}^{*}\right)\right|^{-\gamma}\left|h\left(X_{k}^{*}-E X_{k}^{*}\right)\right|^{\gamma} \\
& \leq(\cosh (2 h c)-1)(2 c)^{-\gamma} E\left|X_{k}^{*}-E X_{k}^{*}\right|^{\gamma},
\end{aligned}
$$

and hence

$$
E e^{h\left(S_{n}^{*}-E S_{n}^{*}\right)} \leq \exp \left\{(\cosh (2 h c)-1)(2 c)^{-\gamma} B_{n, \gamma}\right\}
$$

In the same way, one can prove the inequality

$$
E e^{-h\left(S_{n}^{*}-E S_{n}^{*}\right)} \leq \exp \left\{(\cosh (2 h c)-1)(2 c)^{-\gamma} B_{n, \gamma}\right\}
$$

From these inequalities and from (3.11), it follows that

$$
\begin{aligned}
P\left\{\max _{1 \leq k \leq n}\left|S_{k}-E S_{k}\right|>x\right\} & \leq 4 \exp \left\{-h x+(\cosh (2 h c)-1)(2 c)^{-\gamma} B_{n, r}\right\} \\
& \leq 4 \exp \left\{-h x+2\left(e^{2 h c}-1\right)(2 c)^{-\gamma} B_{n, r}\right\} .
\end{aligned}
$$

Put $h=(2 c)^{-1} \ln \left(x c^{\gamma-1} / B_{n, \gamma}+1\right)$. As a result we obtain (3.2).

Lemma 3.2. Let $h(x), x \in[0, \infty)$, be a nondecreasing nonnegative function, $\xi$ be a nonnegative random variable, $r \geq 1$, and $q>0$. Then

$$
\sum_{n=1}^{\infty} n^{r-1} h(n) P\left\{\xi>n^{1 / q}\right\} \leq E\left(\xi^{r q} h\left(\xi^{q}\right)\right) .
$$

Proof. It can be found in Kruglov and Volodin [7]. 
Lemma 3.3. Let $h(x), x \in[0, \infty)$, be a nondecreasing nonnegative function possessing property (2.1), $\xi$ be a nonnegative random variable, $r \geq 1, q>0$, and $\left\{b_{n}\right\}_{n \geq 1}$ an unbounded nondecreasing sequence of positive numbers such that $b_{n+1} \leq b b_{n}$ for all $n \in \mathbb{N}$ and for some number $b>0, b_{0}=0$. Then there exist numbers $k_{0} \in \mathbb{N}$ and $d>0$ such that

$$
\left.d E\left(\xi^{r q} h\left(\xi^{q}\right)\right) I_{\left\{\xi \geq b_{k_{0}}\right\}}\right) \leq \sum_{n=1}^{\infty} b_{n}^{r-1} h\left(b_{n}\right)\left(b_{n}-b_{n-1}\right) P\left\{\xi>b_{n}^{1 / q}\right\}
$$

Proof. It can be found in Kruglov and Volodin [7].

The next lemma was proved in Kruglov and Volodin [7] under an additional restriction.

Lemma 3.4. Let $X_{n}, n \in \mathbb{N}$, be identically distributed random variables such that $E\left|X_{1}\right|^{q}<\infty$ for some $q \in(0,2)$ and $E X_{1}=0$ if $1 \leq q<2$. Define the function $f(x)=-\varepsilon n^{1 / q} I_{\left(-\infty,-\varepsilon n^{1 / q}\right)}(x)+$ $x I_{\left[-\varepsilon n^{1 / q}, \varepsilon n^{1 / q}\right]}(x)+\varepsilon n^{1 / q} I_{\left(\varepsilon n^{1 / q}, \infty\right)}(x), x \in \mathbb{R}$, where $\varepsilon>0$ is a fixed number. Then

$$
\lim _{n \rightarrow \infty} \frac{1}{n^{1 / q}} \max _{1 \leq k \leq n}\left|\sum_{j=1}^{k} E f\left(X_{j}\right)\right|=0 .
$$

Proof. Note that

$$
\begin{gathered}
\max _{1 \leq k \leq n}\left|\sum_{j=1}^{k} E f\left(X_{j}\right)\right|=n\left|E f\left(X_{1}\right)\right| \leq n\left|E\left(X_{1} I_{\left\{\left|X_{1}\right| \leq \varepsilon n^{1 / q}\right\}}\right)\right|+\varepsilon n^{1 / q} P\left\{\left|X_{1}\right|>\varepsilon n^{1 / q}\right\} \\
\lim _{n \rightarrow \infty} \frac{1}{n^{1 / q}} n^{1 / q+1} P\left\{\left|X_{1}\right|>\varepsilon n^{1 / q}\right\}=\lim _{n \rightarrow \infty} n P\left\{\left|X_{1}\right|^{q}>\varepsilon^{q} n\right\}=0 .
\end{gathered}
$$

It suffices to prove that

$$
\lim _{n \rightarrow \infty} \frac{n}{n^{1 / q}} E\left(X_{1} I_{\left\{\left|X_{1}\right| \leq \varepsilon n^{1 / q}\right\}}\right)=0
$$

Suppose that $0<q<1$. For any $\delta>0$, there exist $n_{0} \in \mathbb{N}$ such that $E\left(\left|X_{1}\right|^{q} I_{\left\{\left|X_{1}\right|>\varepsilon n^{1 / q\}}\right.}\right)<\delta$. We get, for any $n \geq n_{0}$,

$$
\frac{n}{n^{1 / q}} E\left(\left|X_{1}\right| I_{\left\{\left|X_{1}\right| \leq \varepsilon n^{1 / q}\right\}}\right) \leq \frac{n}{n^{1 / q}} E\left(\left|X_{1}\right| I_{\left\{\left|X_{1}\right| \leq \varepsilon n_{0}^{1 / q}\right\}}\right)+\varepsilon^{1-q} E\left(\left|X_{1}\right|^{q} I_{\left\{\varepsilon n_{0}^{1 / q}<\left|X_{1}\right| \leq \varepsilon n^{1 / q}\right\}}\right),
$$

and hence

$$
\limsup _{n \rightarrow \infty} \frac{n}{n^{1 / q}} E\left(\left|X_{1}\right| I_{\left\{\left|X_{1}\right| \leq n^{1 / q}\right\}}\right) \leq \varepsilon^{1-q} \delta
$$

This implies (3.21), since $\delta>0$ can be chosen arbitrarily small. 


$$
\begin{aligned}
& \text { If } 1 \leq q<2 \text { and } E X_{1}=0 \text {, then we get } \\
& \frac{n}{n^{1 / q}}\left|E\left(X_{1} I_{\left\{\left|X_{1}\right| \leq \varepsilon n^{1 / q\}}\right.}\right)\right|=\frac{n}{n^{1 / q}}\left|E\left(X_{1} I_{\left\{\left|X_{1}\right|>\varepsilon n^{1 / q}\right\}}\right)\right| \leq \varepsilon^{1-q} E\left(\left|X_{1}\right|^{q} I_{\left\{\left|X_{1}\right|>\varepsilon n^{1 / q}\right\}}\right) \longrightarrow 0 .
\end{aligned}
$$

\section{Acknowledgments}

The author is grateful to an anonymous referee for careful reading of the paper and useful remarks. This research was supported by the Russian Foundation for Basic Research, projects 08-01-00563-a, 08-01-00567-a, 08-01-90252 Uzbekistan.

\section{References}

[1] P. L. Hsu and H. Robbins, "Complete convergence and the law of large numbers," Proceedings of the National Academy of Sciences of the United States of America, vol. 33, pp. 25-31, 1947.

[2] P. Erdös, "On a theorem of Hsu and Robbins," Annals of Mathematical Statistics, vol. 20, pp. 286-291, 1949.

[3] P. Erdös, "Remark on my paper "On a theorem of Hsu and Robbins", " Annals of Mathematical Statistics, vol. 21, article 138, 1950.

[4] F. Spitzer, "A combinatorial lemma and its application to probability theory," Transactions of the American Mathematical Society, vol. 82, pp. 323-339, 1956.

[5] L. E. Baum and M. Katz, "Convergence rates in the law of large numbers," Transactions of the American Mathematical Society, vol. 120, pp. 108-123, 1965.

[6] V. M. Kruglov, A. I. Volodin, and T.-C. Hu, "On complete convergence for arrays," Statistics and Probability Letters, vol. 76, no. 15, pp. 1631-1640, 2006.

[7] V. M. Kruglov and A. I. Volodin, "Convergence rates in the law of large numbers for arrays," Probability and Mathematical Statistics, vol. 26, no. 1, pp. 63-76, 2006.

[8] R. L. Taylor, R. F. Patterson, and A. Bozorgnia, "A strong law of large numbers for arrays of rowwise negatively dependent random variables," Stochastic Analysis and Applications, vol. 20, no. 3, pp. 643$656,2002$.

[9] P. Chen, N.-C. Yu, X. Liu, and A. Volodin, "On complete convergence for arrays of rowwise negatively associated random variables," Theory of Probability and Its Application, vol. 52, pp. 393-397, 2007.

[10] P. Chen, N.-C. Yu, X. Liu, and A. Volodin, "On complete convergence for arrays of rowwise negatively associated random variables," Probability and Mathematical Statistics, vol. 26, pp. 63-76, 2007.

[11] Q.-M. Shao, "A comparison theorem on moment inequalities between negatively associated and independent random variables," Journal of Theoretical Probability, vol. 13, no. 2, pp. 343-356, 2000.

[12] Yu. V. Prohorov, "Some remarks on the strong law of large numbers," Theory of Probability and Its Applications, vol. 4, pp. 215-220, 1959.

[13] D. H. Fuk and S. V. Nagaev, "Probabilistic inequalities for sums of independent random variables," Theory of Probability and Its Applications, vol. 16, pp. 660-675, 1971.

[14] V. M. Kruglov, "Strengthening the Prokhorov arcsine inequality," Theory of Probability and Its Applications, vol. 50, no. 4, pp. 767-774, 2005. 


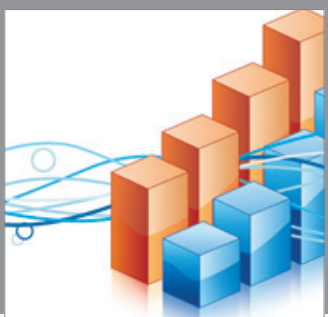

Advances in

Operations Research

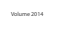

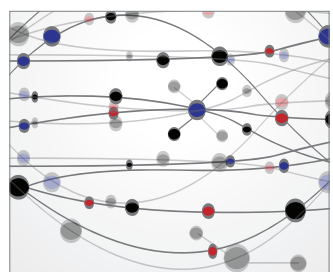

\section{The Scientific} World Journal
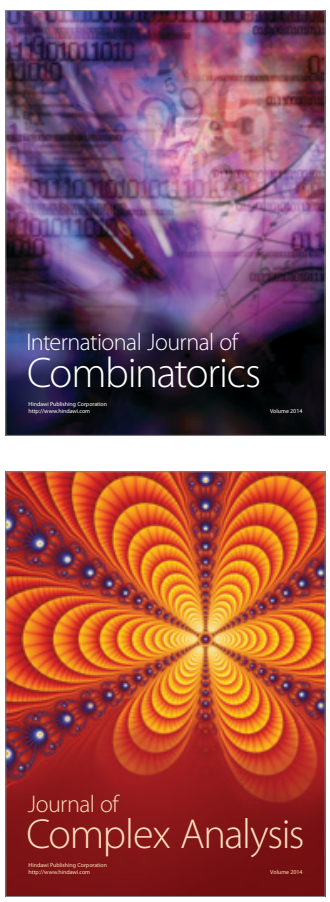

International Journal of

Mathematics and

Mathematical

Sciences
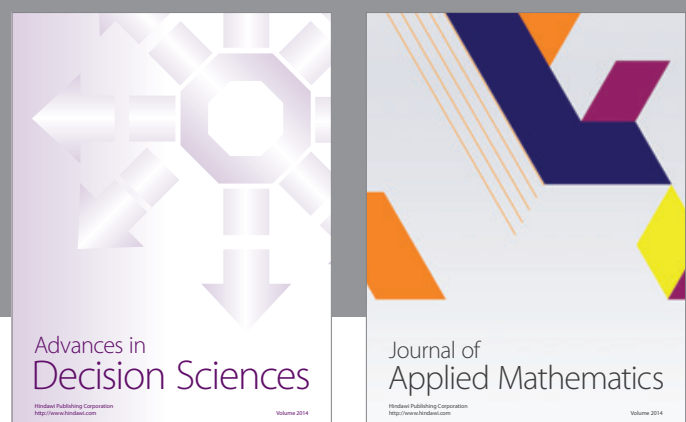

Journal of

Applied Mathematics
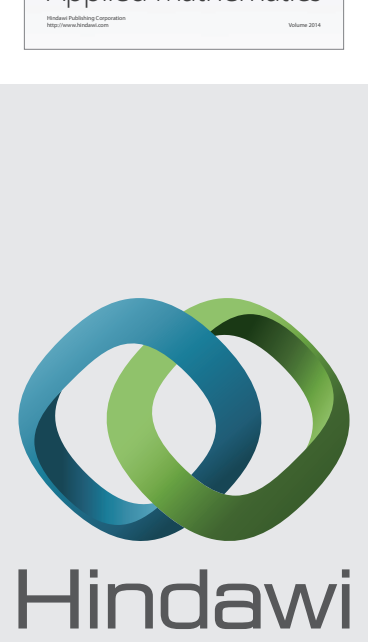

Submit your manuscripts at http://www.hindawi.com
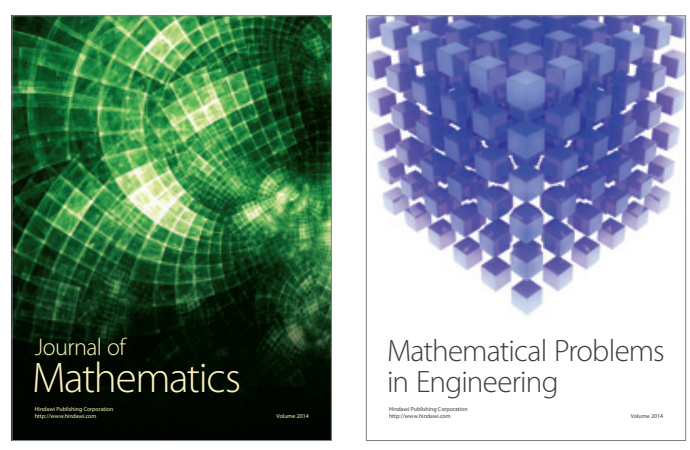

Mathematical Problems in Engineering
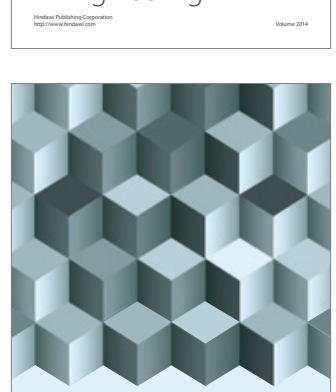

Journal of

Function Spaces
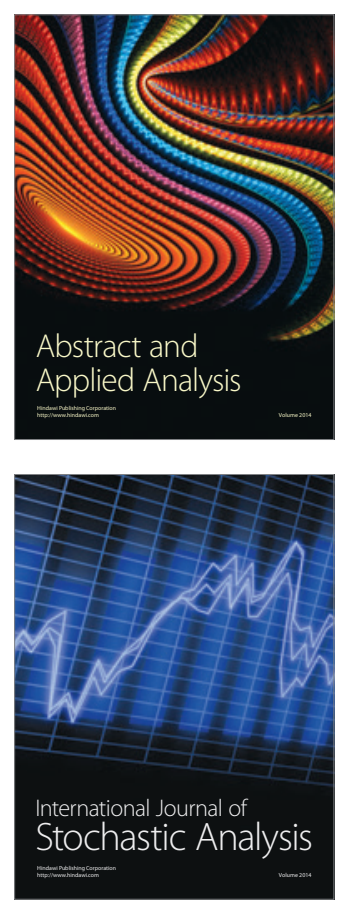

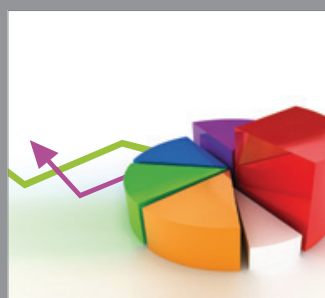

ournal of

Probability and Statistics

Promensencen
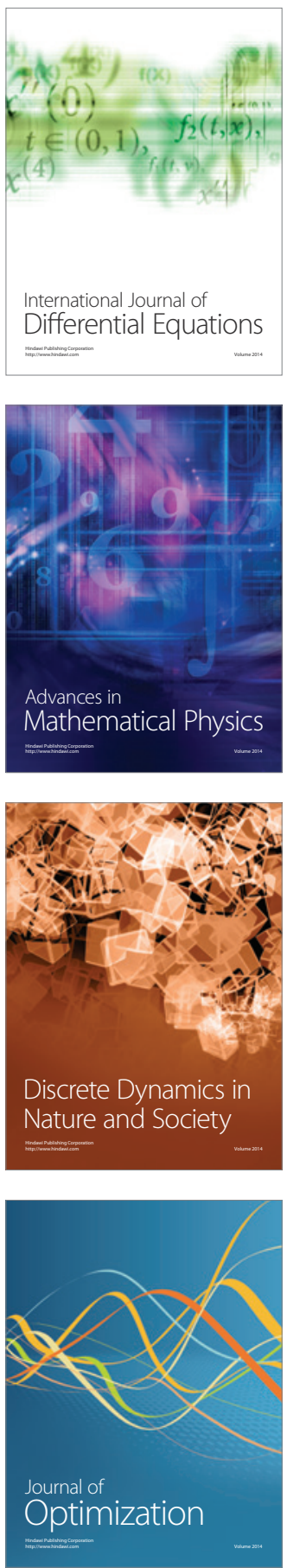